\title{
Anabases
}

ANABASES Traditions et réceptions de l'Antiquité

22 | 2015

Varia

« Et la Grèce le scella de son empreinte » Pierre Paris, des lettres à l'archéologie, du Normalien à l'Athénien

\section{Grégory Reimond}

\section{OpenEdition}

Journals

Édition électronique

URL : http://journals.openedition.org/anabases/5490

DOI : $10.4000 /$ anabases.5490

ISSN : 2256-9421

Éditeur

E.R.A.S.M.E.

\section{Édition imprimée}

Date de publication : 20 octobre 2015

Pagination : 167-192

ISSN : 1774-4296

\section{Référence électronique}

Grégory Reimond, « «Et la Grèce le scella de son empreinte »Pierre Paris, des lettres à l'archéologie, du Normalien à l'Athénien », Anabases [En ligne], 22 | 2015, mis en ligne le 20 octobre 2018, consulté le 21 octobre 2019. URL : http://journals.openedition.org/anabases/5490; DOI : 10.4000/anabases. 5490 


\section{"Et la Grèce le scella de son empreinte ${ }^{1}$ » Pierre Paris, des lettres à l'archéologie, du Normalien à l'Athénien²}

Grégory ReImond

$S^{\prime}$ il est un pays auquel le nom de Pierre Paris (I859-I93i) reste attaché, c'est bien l'Espagne. Figure majeure de l'hispanisme archéologique, précurseur des études sur la culture ibérique qu'il contribue à faire connaître - et reconnaître - à la suite de Léon $\mathrm{Heuzey}^{3}$, son nom est lié à un événement qui va marquer le cours de sa carrière: l'achat du célèbre buste de la Dame d'Elche qu'il réalise pour le compte du musée du Louvre en I897 ${ }^{4}$. Sa voie est alors tracée. Jusqu'à sa mort, le savant bordelais travaille sans relâche à mieux connaître le pays qu'il a adopté, tout en suscitant un grand nombre d'études sur l'histoire et la

1 En référence à G.RADEt, L'histoire et l'œuvre de l'École française d'Athènes, Paris, A. Fontemoing, I9oI, p. 422.

2 Ce travail s'inscrit dans le cadre du projet de recherche HAR 20I2-3I 36 «Antigüedad, nacionalismos e identidades complejas en la historiografía occidental (I700-I90o): los casos español, británico y argentino ", sous la direction d'Antonio Duplá (université du Pays basque).

3 Voir notamment, “L’archaïsme gréco-phénicien en Espagne», CRAI I89o, p.125-128, “Statues espagnoles de style gréco-phénicien», $B C H$ i5, I89i, p.6o8-625 et “Mission de M. Pierre Paris en Espagne. Note de M.Heuzey, membre de l'Académie », CRAI I897, p.5o5-5og.

4 Parmi une abondante bibliographie, retenons l'étude de C. PAPí Rodes, “La venta de la Dama de Elche. Desmontando algunas justificaciones ", Recerques del Museu d'Alcoi i4, 2005, p. 157-168, ainsi que R. Olmos \& T. Torтosa (éd.), La Dama de Elche. Lecturas desde la diversidad, Madrid, Lynx, Agepasa, ig97 et A. Rodero Riaza (dir.), Cien años de una Dama, Madrid, Ministerio de Educación y cultura, I997. 
culture hispaniques à travers des institutions dont il est en grande partie le fondateur (l'École des hautes études hispaniques puis la Casa de Velázquez) ${ }^{5}$.

Pierre Paris reste pourtant une figure méconnue. Si les notices biographiques les plus récentes consacrent l'essentiel de leur développement aux “années ibériques ${ }^{6}$ », nous avons pu constater à quel point le Pierre Paris “d'avant l'Espagne » avait été négligé par ses biographes. Ces derniers se contentent de rappeler, en guise d'introduction et comme prélude à une carrière d'hispaniste qui allait de soi, son passage à l'École normale supérieure (I879-I882), son succès à l'agrégation de lettres (I882), son séjour à l'École française d'Athènes (I882-I885), enfin son entrée dans le monde universitaire dès son retour de Grèce. Il est alors maître de conférence à l'université de Bordeaux avant d'occuper, en I892, la chaire d'archéologie et d'histoire de l'art. Rares sont les historiens à s'être intéressés aux différentes étapes de ce brillant parcours. Seul Jean Marcadé nous donne de précieux détails sur cette période de la vie de Pierre Paris ${ }^{7}$. Ce constat

5 Les études relatives à l'histoire de l'institutionnalisation des études hispaniques n'ont cessé de se multiplier ces dernières années. Retenons notamment J.-M. Delaunay, Des palais en Espagne. L'École des hautes études hispaniques et la Casa de Velázquez au coeur des relations franco-espagnoles du xx siècle (I898-1979), Madrid, Casa de Velázquez, I994; P. Moret \& P. Cressier, “La Casa de Velázquez y la arqueología: algunos apuntes históricos », in M. B.Deamos, J. Beltrán Fortes (éd.), Las instituciones en el origen y desarrollo de la arqueología en España, Sevilla, spal monografías ıo, Universidad de Sevilla, 2007, p.343-36o. Plus largement, A. Niño Rodríguez, Cultura y diplomacia. Los hispanistas franceses y España (I875-I93I), Madrid, csic, Casa de Velázquez, Société des hispanistes français, 1988 .

6 Voir notamment P. Moret, “Pierre Paris (I859-I93i), précurseur de l'archéologie ibérique », in C. Aranegui, J.-P.Mohen, P. Rouillard, C. Eluère (éd.), Les Ibères [catalogue de l'exposition, Paris, Galeries nationales du Grand Palais - Barcelone, Fundación “La Caixa» - Bonn, Kunst- und Ausstellungshalle der Bundesrepublik Deutschland], Dijon, Faton, I997-I998, p. 70-7I; G. Mora, “Pierre Paris y el hispanismo arqueológico », Anejos de Archivo español de arqueología 3o, 2004, p.27-42; P. Roulllard, "Pierre Paris », in M. Ayarzagüena Sanz, G. Mora (éd.), Pioneros de la arqueología en España (del siglo XVI a IgI2), Alcalá de Henares, Museo Arqueológico Regional, 2004, p.3II-3I5, et plus récemment, “Paris, Pierre (I5 janvier I859, Rodez - 2ooctobre I93I, Madrid)», in P.Sénéchal \& C.Barbillon (dir.), Dictionnaire critique des historiens de l'art actifs en France de la Révolution à la Première Guerre mondiale, Paris, Site web de l'INHA, 2009, [http://www.inha.fr/spip.php?article248o], consulté le 3rjuillet 20I3, ainsi que la notice qui lui est consacrée dans M. Díaz-Andreu, G. Mora \& J. Cortadella (éd.), Diccionario histórico de la arqueología en España, Madrid, Marcial Pons, 2009, p.5ı-5ı.

7 “La vie et l'œuvre de Pierre Paris », in Célébration du centenaire de la naissance de Pierre Paris et de Georges Radet (Io et II mars Ig6I), Bordeaux, Faculté des lettres et sciences humaines, ig63, p. I4-3o. 
nous a incité à pousser plus avant nos recherches sur les premières années de formation du jeune savant.

Les résultats de ce travail se sont révélés étonnamment riches et engagent plusieurs axes d'étude. Le premier concerne l'action et l'œuvre de l'Athénien; viennent ensuite les années passées à l'université de Bordeaux, qui sont celles de l'entrée dans la vie professionnelle, des premières publications d'envergure et de la participation à des projets collectifs (comme la rédaction du Dictionnaire des antiquités grecques et romaines); enfin, la question de sa progressive conversion à l'hispanisme, restée jusque-là inexpliquée et pourtant essentielle dans la perspective d'une biographie intellectuelle de Pierre Paris. L'argument avancé par Jean-Marc Delaunay et repris par d'autres n'est en effet pas recevable: la situation politique de la Grèce et de l'Empire ottoman au tournant du xix ${ }^{\mathrm{e}}$ et du Xx $\mathrm{xx}^{\mathrm{e}}$ siècle ne suffit pas à expliquer que Pierre Paris se soit détourné des études grecques pour porter son regard vers la péninsule ibérique ${ }^{8}$.

Dans le présent essai, nous nous proposons d'explorer les raisons qui ont orienté Pierre Paris vers l'archéologie. Voilà une première conversion qu'il convient d'expliquer, en mettant en lumière les différentes étapes qui conduisent Pierre Paris à faire le choix d'une science en pleine formation, et par là même pleine de promesses.

\section{À «l'école de précision de l'esprit français »" le Normalien de la rue d'Ulm}

Le 27 octobre I882 est publié l'arrêté de nomination faisant de Pierre Paris l'un des deux membres de la 33e promotion de l'École française d'Athènes ${ }^{10}$ (EFA). Jeune agrégé de lettres tout juste sorti de l'École normale supérieure (ENs), il n'a pas encore fêté ses 23 ans mais son parcours est déjà brillant. Si cette ascension n'est pas déterminée par le milieu dans lequel Pierre Paris a grandi, l'étude de

8 J.-M. Delaunay i994, p.23. Comment pourrions-nous expliquer alors le dynamisme et la vitalité des activités déployées par l'École française d'Athènes à la même époque? Rappelons simplement que les années I892-Igo3 sont celles de la grande fouille de Delphes, tandis que l'EFA explore Argos entre I902 et I9I3; grâce au mécénat du duc de Loubat, les fouilles reprennent à Délos en igo3.

9 Le mot serait de Désiré Nisard (I806-1888), directeur de l'École normale supérieure (I857-I867). À l'impératrice Eugénie qui lui aurait demandé ce qu'était l'école qu'il dirigeait, Nisard aurait répondu: «Madame, l'École normale, c'est l'école de précision de l'esprit français». L'anecdote est rapportée dans G. Lanson, L'École normale supérieure, Paris, Hachette, I926, p. 32.

10 Le deuxième nom retenu est celui de Maurice Holleaux (I86I-I932). Cf. È. GranAymerich, Les chercheurs de passé. I798-I945. Aux sources de l'archéologie, Paris, cnns, 2007, p. 875-877 (il s'agit de la notice de son Dictionnaire biographique d'archéologie). 
ses origines sociales permet toutefois de l'éclairer. On constate alors que le jeune Athénien avait toutes les chances d'intégrer le groupe d'élite auquel s'ouvrent les portes des grandes écoles françaises de la III République, destinées à «faire surgir, dans la masse qui n'attend de l'université que l'accès à une profession, le petit nombre de ceux qui pourront consacrer leur vie à des "recherches savantes "1"”.

Son passage par la rue d'Ulm (I879-I882) montre que Pierre Paris appartient à une minorité de privilégiés bénéficiant de la meilleure formation, celle qui ouvre la voie aux filières d'excellence des grandes écoles, réservées à la bourgeoisie fût-elle petite et provinciale ${ }^{12}$. En ce sens, son succès au concours d'entrée à l'ENs s'avère décisif pour la suite de sa carrière. Il lui permet de franchir « la première étape de la sélection dans l'ascension professionnelle à une époque où «le passage à l'École normale s'avère le critère stratificateur de l'Université qui en commande, dans une certaine mesure, tous les autres ${ }^{13}$ ». Pierre Paris débute en effet sa carrière dans les années I88o, une décennie qui voit “l'apogée triomphale de la société Normalienne ${ }^{14}$ ». Le séjour à l'Efa constitue la seconde étape de cette ascension, indissociable de son statut de Normalien. En effet, si depuis le décret du 7 août I85o les membres de l'EFA ne sont plus nécessairement choisis parmi les agrégés sortis de l'Ens, Catherine Valenti rappelle qu'entre i846 et ig9o, sur les 242 membres admis, seuls 33 d'entre eux (un peu moins de I4\%) ne sont pas passés par l'école de la rue d'Ulm ${ }^{15}$. Enfin, la troisième étape de ce cursus honorum est franchie dès I885, lorsque Pierre Paris obtient son premier poste à l'université de Bordeaux, dès son retour de Grèce. Or en I8go, il y a seulement un professeur de faculté non Normalien pour trois professeurs Normaliens de la rue d'Ulm ${ }^{16}$. Les trois années passées à l'ENs ont donc à la fois favorisé et accéléré la carrière de Pierre Paris.

L’origine géographique et sociale est un autre élément à prendre en compte pour expliquer sa réussite professionnelle. Né le i5 janvier i859 à Rodez, dans l'Aveyron, il est issu de la petite bourgeoisie «intellectuelle» et “cultivée» provinciale

1 A. Renaut, “Une philosophie française de l'université allemande. Le cas de Louis Liard ", Romantisme 88, 1995, p. 95 .

12 Rappelons qu'à la fin du XIx ${ }^{\mathrm{e}}$ siècle, le taux de sélection à l'ens est de l'ordre de I sur Io pour les littéraires. Cf. V.KARADY, “Normaliens et autres enseignants à la Belle Époque. Note sur l'origine sociale et la réussite dans une profession intellectuelle», Revue française de sociologie $\mathrm{I} 3$ (I), I972, p. 36 (note 4).

13 V. KaRADY, “L'expansion universitaire et l'évolution des inégalités devant la carrière d'enseignant au début de la III République», Revue française de sociologie I4 (4), I973, p. $453-454$.

14 Ibid., p. 466.

15 C. VAlENTI, «Les membres de l'École française d'Athènes : étude d'une élite universitaire (I846-I992) », BCH I20 (I), I996, p. I57-I72.

16 V. Karady 1973, p. 466. 
pour laquelle une carrière dans l'enseignement est un des principaux vecteurs de la mobilité sociale ${ }^{17}$. Comme l'indique son acte de naissance ${ }^{18}$ (Fig. I), son père, Marie Gustave Léon Paris, est en effet professeur au lycée Impérial de Rodez (l'actuel lycée Foch). Or si le professorat fournit seulement 6,5\% des enseignants dans les années I870, 3I,4\% des Normaliens en sont issus, ce qui en fait la catégorie socioprofessionnelle la plus représentée ${ }^{19}$. Pierre Paris est ainsi issu d'un milieu où les chances d'accès à l'ENs (dont la mission est d'abord de former des agrégés et donc de futurs enseignants) sont les plus fortes. Le facteur géographique n'est pas à négliger. Victor Karady a démontré la sur-représentation de la population urbaine parmi les Normaliens ${ }^{20}$ : entre I87I et I9I4, I8,4\% d'entre eux sont nés à Paris, 3I,9\% dans un chef-lieu de département, ce qui est le cas pour Pierre Paris. La précision pourrait sembler anecdotique. Elle traduit pourtant la possibilité d'accéder à la fois à l'enseignement secondaire «bourgeois » dispensé dans les lycées classiques, et aux différentes formes de cultures offertes dans les centres urbains. C'est là une condition essentielle sinon indispensable pour qu'un jeune provincial puisse intégrer les filières d'excellence proposées par les grands établissements parisiens, des classes préparatoires à l'ENs ${ }^{21}$. Les chiffres avancés par Catherine Valenti'22 dans son étude sur les Athéniens confirment ceux de Victor Karady sur les Normaliens (Fig. 2) : Pierre Paris voit le jour dans une région qui est loin d'être un vivier d'Athéniens. Entre i846 et I990, le Sud-Ouest ne fournit que 8\% des membres de l'EFA, loin derrière le Nord-Est ou Paris et sa région qui totalisent respectivement $28 \%$ et $27 \%$ de l'effectif. Cependant, son origine géographique le rattache également aux $24 \%$ des membres nés dans un chef-lieu de département, et son origine sociale aux 22 \% d'Athéniens Normaliens fils de professeurs de l'enseignement secondaire (la catégorie socio-professionnelle la plus représentée). Les origines géographiques et sociales de Pierre Paris doivent donc être mises en relation avec son ascension professionnelle: elles lui ont permis d'accroître ses chances de réussite dans le système de la méritocratie républicaine. Fort de ce capital socio-culturel, sans doute poussé par sa famille désireuse de lui assurer un brillant avenir, peut-être repéré par un inspecteur académique ou un proviseur de lycée, c'est dans la capitale qu'il achève ses études secondaires. Quitter sa terre natale est vécu comme un déracinement, un sentiment sans doute accentué par l'éloignement familial et l'expérience de l'internat: “Triste écolier de quatrième exilé loin du pays familier

17 V. Karady 1972, p.37.

18 Il est conservé aux Archives départementales de l'Aveyron: 4E2I2-38 RODEZ NA I859/ı859 - (oI.oI.I859 - 28.oI.I859).

19 Ibid., p.4I-42.

20 Ibid., p. 46.

21 Ibid., p.49.

22 C. VAlEnti, I996. 
vers les rigueurs d'un sombre collège parisien ${ }^{23}$ », il poursuit ses études au collège Sainte-Barbe, “au moment où ce collège était dans tout son éclat ${ }^{24}$ ». Par la suite, c'est au lycée Louis-le-Grand que Pierre Paris prépare son concours d'entrée à l'École normale supérieure ${ }^{25}$. En 1879 , il a tout juste 20 ans lorsqu'il franchit le portail de l'école de la rue d'Ulm.

Toutefois, le séjour de Pierre Paris à l'Ens représente bien plus qu'un simple levier permettant de favoriser et d'accélérer sa carrière. L'ENs lui offre avant tout la possibilité de se former à des méthodes nouvelles propres à «l'esprit normalien ${ }^{26}$ ». Une formation qui reflète les derniers acquis de la science, notamment historique, fondée désormais sur une méthode rigoureuse, une approche critique des textes et des sources en général, la volonté d'adopter une véritable démarche scientifique (héritée des érudits de la monarchie de Juillet et du Second Empire, inspirée de l'œuvre des savants allemands). Or dans les années I87o, cette “école méthodique» en formation est absente des facultés françaises. On ne la trouve guère qu'au sein des “écoles spéciales» que sont l'Ens, l'École polytechnique, l’École pratique des hautes études, de fondation récente, le Collège de France ou encore l'École des Chartes ${ }^{27}$. Comme le rappelle Gustave Lanson dans la monographie qu'il publie sur l'ENs en I926, “toutes les curiosités, toutes les vocations peuvent

23

J. Marcadé, 1963, p. I4.

A. Puech, «Pierre Paris », crai I93i, p.334.

Entre i87i et I9ı4, 65\% des Normaliens littéraires préparent leur concours à Louisle-Grand ou Henri-IV (V.Karady I972, p.49, note 23). Comme Pierre Paris, $43 \%$ des Athéniens qui ont préparé ce concours à Paris l'ont fait à Louis-le-Grand (C. VAlEnti I996, p. I58).

G. Lanson, I926, p.3o.

La bibliographie sur ce thème est abondante. Nous renvoyons ici aux références qui nous paraissent essentielles: G.Bourdé \& H.Martin, Les écoles historiques, Paris, Seuil, Points histoire, I983, (rééd. 1997); C. Delacroix, F. Dosse \& P. Garcia, Les courants historiques en France. XIX $-X X^{e}$ siècle, Paris, Gallimard, 2007; A.Prost, Douze leçons sur l'histoire, Paris, Seuil, Points histoire, I996. Voir également C.-O. CARbonell, “L'histoire dite "positiviste" en France», Romantisme 8 (2I), І978, p. I73-г85; А.Рвоsт, “Seignobos revisité», Vingtième siècle. Revue d'histoire 43, I994, p. IOо-II8; J.Revel, "Histoire et sciences sociales. Lecture d'un débat français autour de I9oo ", Mil neuf cent 25, 2007, p. IоI-I26. С. Seignobos, “L'enseignement de l'histoire comme instrument d'éducation politique», in Conférences du Musée pédagogique. L'enseignement de l'histoire, Paris, Imprimerie nationale, I907, p. I-24, réédité et présenté par A.Prost, Vingtième siècle. Revue d'histoire 2 (I), I984, p. Io3-Io8; F. Simiand, “Méthode historique et science sociale. Étude critique d'après les ouvrages récents de M.Lacombe et de M. Seignobos ", Revue de synthèse historique 6 (I), I9o3, p.I-22, repris dans les Annales I5 (I), I96o, p.83-ı. Concernant la place de l'archéologie dans ce contexte, voir l'indispensable synthèse d'È. Gran-Aymerich 2007. 
s'éveiller et s'armer dans la maison de la rue d'Ulm; tout ce qui s'apprend, tout ce qui s'enseigne, tout ce qui est matière de recherche critique et de construction méthodique y trouve place. [...] II est certain que la formation philosophique et scientifique a toujours tenu plus de place à l'École que la formation pédagogique et cela a pu fournir à certains individus les ressources intellectuelles qui leur ont permis de chercher fortune hors de l'Université. [...] On s'occupe d'abord de faire des lettrés, des érudits et des savants solidement armés et l'on fait ensuite des professeurs ${ }^{28}$ ).

Cette pépinière de savants est aussi un puissant lieu de sociabilité, un espace de discussions et d'échanges, animé par des enseignants aguerris et de brillants étudiants, ouverts aux débats les plus divers et les plus actuels, où se pratique une véritable maïeutique. En somme, un lieu où l'on ne se contente pas de faire des têtes bien pleines mais où l'on forme également des têtes bien faites :

"Toutes les opinions sont libres, libres d'être aussi extrêmes, absolues et violentes qu'il leur plaît, mais chacune accorde aux autres le droit qu'elle réclame pour ellemême. Les discussions sont passionnées et la tolérance entière. Il y a là une tradition saine et forte qu'aucune influence mauvaise des mœurs du dehors n'a encore réussi à entamer. Avec ce goût de liberté et cette habitude de tolérance, le caractère le plus marqué de l'esprit normalien me paraît consister dans une prédominance de l'intelligence sur l'imagination et la sensibilité. Le besoin de comprendre, de définir, d'expliquer est le besoin essentiel; l'horreur du vague, de l'obscur, de l'incohérent est poussée au dernier point. [... L'enseignement de l'École a toujours été fondé sur le libre examen, la libre discussion. Les Conférences devaient être, selon l'esprit de leur institution, et n'ont jamais cessé complètement d'être des entretiens du maître et des élèves; et, comme le disait M. Georges Perrot en i895, "admettre et même inviter les élèves à discuter avec le professeur, c'est reconnaître que dans l'ordre de la pensée il n'y a point d'autorité, fût-ce celle du maître le plus savant et le plus respecté, qui ne soit tenue de donner ses raisons et de fournir ses preuves". S'il en est ainsi entre élèves et maîtres, on peut deviner ce qu'il en sera entre camarades ${ }^{29}$.

Or lorsque l'on regarde le nom des condisciples de Pierre Paris, membres de sa promotion (I879) ou des promotions voisines, on imagine aisément la richesse de ces discussions et leur apport à sa formation intellectuelle: Émile Durkheim, Ferdinand Brunot, René Grousset, Paul Fabre, René Doumic, Maurice Holleaux pour la promotion de Pierre Paris, celle de i879; Joseph Bilco, Michel Clerc, Camille Jullian, Alfred Rébelliau, Raymond Thamin pour celle de ı877; Paul Monceaux, Alphonse Veyries, Charles Diehl, Alfred Baudrillart, Henri Bergson,

28 G. Lanson, I926, p.2I et 27. Il est lui-même un ancien élève de la promotion de i876 et dirige l'école entre igrg et I927.

29 G. Lanson, I926, p. 3I-32 et 34. 
Paul Desjardins, Jean Jaurès pour celle de ı878; Georges Cousin, Félix Dürrbach, Étienne Dejean, Pierre Imbart de la Tour rejoignent quant à eux l'École en i88o, Georges Radet, Henri Berr ou Lucien Gallois en i88ı. D’une certaine manière, on peut considérer que ces années parisiennes lui permettent de jeter les bases d'un véritable réseau qui aura son importance dans la suite de sa carrière, d'autant que, si l'on en croit Aimé Puech, “avec ses dons heureux et spontanés, il s'était bien vite fait de tous ses camarades des amis ${ }^{30}$ ». Il tisse avec certains des liens étroits, notamment Georges Radet, avec lequel Paris est uni par «les liens d'une fraternelle amitié31 », Maurice Holleaux, son compagnon Athénien de la 33e promotion, ou encore Camille Jullian, Édouard Cuq, Émile Vigneaux et Georges Radet luimême qui seront ses collègues à l'université de Bordeaux.

Le séjour à l'EFA permet de resserrer ces liens ou de les étendre. Pierre Paris y retrouve un grand nombre de ses anciens camarades de la rue d'Ulm, ou y fait la connaissance de personnalités comme Arthur Engel, avec lequel il travaillera plus tard en Espagne (Fig. 3): sur les 4r membres des Écoles françaises d'Athènes et de Rome entre I878 et I884, 22 sont d'anciens Normaliens, soit près de $54 \%$ (tous membres de promotions voisines); pour les seuls Athéniens, leur part atteint près de $87 \%$ de l'effectif (ı3 étudiants sur i5). Ces liens étroits tissés au cours des cinq années passées à Paris puis à Athènes et Rome ne sont pas rompus avec l'entrée des jeunes savants dans la vie professionnelle. Au moins en ce qui concerne ceux qui font carrière dans la recherche et l'enseignement supérieur, ces réseaux continuent à fonctionner. C'est ce que laisse supposer la liste des collaborateurs aux Mélanges Perrot. Recueil de mémoires concernant l'archéologie classique, la littérature et l'histoire anciennes, publiés en Igo3 à l'occasion du $5 \mathrm{o}^{\mathrm{e}}$ anniversaire de l'entrée de Georges Perrot à l'Ens (qu'il dirige de i888 à I902). On y retrouve les noms de Gaston Boissier, Michel Clerc, Amédée Hauvette, Maurice Holleaux, Pierre Paris et Georges Radet, enseignant pour le premier et élèves pour les autres à l'École dans les années I878-I884. Georges Radet ne dit pas autre chose lorsqu'il écrit que l'“ on se sépare; mais on se retrouve. Sans parler du "dîner de Rome et d'Athènes”, où plus tard, à Paris, les liens de camaraderie se resserrent entre contemporains et se créent entre générations différentes, École et Villa tressent par moitié une guirlande de noms indissolubles ${ }^{32}$ ». Il ne nous appartient pas ici d'étudier cette question des réseaux, mais il est évident qu'ils jouent un rôle clé dans la réorientation de la carrière de Pierre Paris vers l'archéologie espagnole.

\footnotetext{
30 A. Puech, I93i, p. 334.

31 J. MarcadÉ, I963, p.4.

32 G. RAdEt, I9OI, p. 247-248.
} 


\section{L'agrégé de lettres (1882)}

L’intérêt de Pierre Paris pour l'archéologie est-il pour autant né « dans la maison de la rue d'Ulm» où, selon Gustave Lanson, “toutes les vocations peuvent s'éveiller ${ }^{33}$ »? Il semble que ce ne soit pas le cas, et l'entrée à l'EFA ne semble pas avoir été d'emblée un objectif pour le jeune Paris, davantage attiré par la philologie classique. C'est ce que laisse entendre Numa Denis Fustel de Coulanges, directeur de l'ENs de I880 à I883, dans une lettre adressée au père de Pierre Paris :

«J'ai parlé à votre fils. J'ai commencé par lui dire que la grammaire n'était pas à dédaigner, et que cette section offrait comme perspective aux jeunes gens $\mathrm{I}^{\circ}$ un bon poste à la sortie de l'École, dans un bon lycée, en seconde ou en troisième; $2^{\circ}$ une belle science à cultiver plus tard. Mais s'il préfère la littérature, il peut dès maintenant signaler ses goûts et ses aptitudes à des professeurs tels que M. Gaston Boissier et M. Petit de Julleville, et il peut être assuré qu'ils ne contrarieront pas sa vocation ${ }^{34}$. »

Il n'est question ici ni d'archéologie, ni même d'histoire. Et si le directeur évoque une possible orientation vers les lettres, “ la belle science à cultiver plus tard » n’arrive qu'en seconde position derrière la perspective d'un «bon poste» dans l'enseignement, propre à assurer un avenir professionnel. Rappelons d'ailleurs que la mission première de l'Ens est de préparer à l'agrégation et au professorat. Cet intérêt pour les lettres transparaît encore dans un discours écrit par Pierre Paris à l'occasion du banquet de la Saint-Charlemagne en $1879^{35}$ :

“Vous méprisez le grec: c’est une indigne chose,

Messieurs, et devant vous je veux plaider sa cause.

Vous n'avez pas le temps! - À quoi donc sert la nuit?

C'est la nuit qu'autrefois en un pauvre réduit,

Éclairant son trésor à la lueur tremblante

Des charbons qu'attisait sa main impatiente,

Amyot, pour oublier le labeur de ses jours,

De Daphnis et Chloé savourait les Amours;

Que le jeune Baï, travailleur héroïque,

Ardent adorateur de la Muse hellénique,

Sans laisser un instant refroidir l'escabeau,

De Ronsard épuisé rallumait le flambeau.

Mais ces mâles vertus ne vous sont pas connues,

Et vous croyez, hélas! Ô races corrompues,

Que la nuit avant tout est faite pour dormir!

G. Lanson, I926, p.2I.

Cité dans J. Marcadé, I963, p. I7.

Le texte de ce discours est rapporté par J. MarCADÉ, I963, p. I6. 
Ah! Ce n'est pas ainsi que l'École Normale

Vous ouvrira jamais sa porte triomphale.»

En dehors de son caractère anecdotique et plaisant, ce texte en vers en dit finalement beaucoup sur le jeune Normalien qu'est Pierre Paris. Tant sur la forme que sur le fond, il témoigne d'un réel attrait pour les lettres, celles de l'Antiquité en particulier. Le discours constitue in fine une ode à la langue grecque, tandis que les références qui le ponctuent évoquent toutes d'éminentes figures de l'érudition humaniste qui ont consacré leur vie à faire connaître les auteurs anciens (comme le traducteur Jacques Amyot) ou à s'en inspirer (comme Jean-Antoine de Baïf et Pierre de Ronsard). Il montre par ailleurs (même si le ton est ironique) que Pierre Paris est conscient, en entrant à l'école de la rue d'Ulm, de s'engager dans une voie difficile et exigeante, faite de dur labeur. Qu'importe, il sacrifiera ses nuits pour tenter de se rapprocher de ces «mâles vertus» qu'il prend pour modèle et pour réussir dans cette voie d'excellence qu'est l'Ens, laquelle vient de lui ouvrir sa “porte triomphale». Trois ans plus tard, en ı882, l'objectif est atteint: Pierre Paris est reçu à l'agrégation de lettres. Il décide alors de se porter candidat pour séjourner à l'EFA. Ce choix, rappelons-le, n'avait rien d'évident. Si l'intérêt de Pierre Paris pour la philologie, les langues anciennes et l'Antiquité est bien réel, l'archéologie reste pour lui un territoire inconnu.

Dès lors, pourquoi l'EFA? Sans doute faut-il y voir l'influence de ses enseignants rue d'Ulm et du directeur de l'école, convaincus de ses capacités. Dans la lettre adressée par celui-ci à son père, Fustel de Coulanges rappelait ainsi “ toute l'estime et toute la sympathie que j'ai pour ce jeune homme. Il a une belle carrière devant lui, et il fera honneur à notre université autant qu'à sa famille ${ }^{36}$ ». Quelle meilleure occasion d'approfondir sa formation, pour un passionné de culture classique, que de séjourner à Athènes, alors que l'École française entre dans l'une de ses plus brillantes périodes sous l'action d'Albert Dumont et de Paul Foucart? Rappelons par ailleurs que Fustel de Coulanges lui-même a été membre de l'EFA ( 9 e promotion de I853), tout comme le professeur de l'École Louis Petit de Julleville ( $7^{\mathrm{e}}$ promotion de i863), tandis que Gaston Boissier s'est illustré comme latiniste et historien de l'Antiquité. Nul doute que la fréquentation de ces maîtres ait incité Pierre Paris à placer ses pas dans les leurs.

Ainsi, par ses origines géographiques et sociales, Pierre Paris se rattache à cette minorité de jeunes gens, issus de la petite bourgeoisie provinciale, ayant accès à la formation classique dispensée dans les établissements secondaires de la III République. À son appartenance à une caste de privilégiés s'ajoutent, comme l'ont souligné ses professeurs, des qualités et des compétences personnelles indéniables qui lui permettent d'accéder à une formation universitaire élitiste et brillante

Cité dans J. MarCadÉ, I963, p. I7. 
(il incarne à ce titre la méritocratie républicaine). Fort de ce parcours sans faute, c'est un Pierre Paris dûment formé aux règles de l'enseignement classique qui est nommé membre de la 33e promotion de l'École française d'Athènes, le 27octobre ı882, aux côtés de Maurice Holleaux.

\section{À l'école d'Albert Dumont et de Paul Foucart}

Les deux nouveaux Athéniens arrivent en Grèce à un moment-clé de l'histoire de l'institution ${ }^{37}$ : sous l'impulsion de ses directeurs successifs, Albert Dumont (1875ı878) et Paul Foucart (ı878-ı89o), sa vocation scientifique s'affirme, l'école devenant un véritable institut de recherche, engagé dans de grandes fouilles concédées par les autorités grecques (notamment à Délos, plus tardivement à Delphes), dans un contexte de forte concurrence avec l'Institut archéologique allemand d'Athènes (fondé en I873). Les règles de fonctionnement de l'EFA sont celles fixées par la réforme de 1874 , menée par Albert Dumont (qui complète la précédente réforme de 1850$)^{38}$. Placée sous le contrôle scientifique de l'Académie des Inscriptions et Belles-Lettres, qui impulse les travaux menés et à laquelle les jeunes Athéniens doivent rendre compte de leurs activités sous la forme de mémoires de recherche ${ }^{39}$, l'école accueille les jeunes savants pour trois ans. Le nouvel Institut de correspondance hellénique (I876) permet, par les séances publiques qu'il organise, de renseigner, d'informer et de stimuler les nouvelles recherches. L'école dispose aussi d'outils pour réunir ces faits et les diffuser. Il s'agit notamment du Bulletin de correspondance hellénique (dont le premier numéro paraît en i877). Plus qu'une simple revue, le Bulletin est un outil pédagogique pour former les jeunes Athéniens: “Grâce à lui, la préparation, la correction de chaque fascicule devint,

37 Sur l'histoire de l'Efa et le contexte scientifique dans lequel se déroule le séjour de Pierre Paris, nous renvoyons à C.VAlenti, L'École française d'Athènes, Paris, Belin, collection “Histoire de l'éducation », 2006; R.ÉTIENnE, “L'École française d'Athènes, I846-I996 ", BCH I20 (I), I996, p.3-22 (l'ensemble du volume est à consulter puisqu'il s'agit d'un numéro spécial paru à l'occasion du cent cinquantenaire de la fondation de l'école); G. Radet igoi ; et È. Gran-Aymerich 2007, notamment p. 203-237 et p.3o2-3o8.

38 Le texte du décret du 26 novembre I874 qui réorganise l'EFA figure en annexe de la monographie de G. RADET, I9oI, p.437-439.

39 Au moins jusqu'en i876, l'Académie des Inscriptions et Belles-Lettres publie ainsi chaque année dans ses Comptes rendus les «questions proposées pour les travaux de l'École française d'Athènes ». Par la suite sont régulièrement publiés des rapports de la Commission des Écoles d'Athènes et de Rome sur les travaux de ces deux Écoles au cours d'une année donnée. Sur les liens entre les deux institutions, voir J. LecLant, “L'École française d'Athènes et l'Académie des Inscriptions et Belles-Lettres: des relations fructueuses au profit des études grecques », $B C H$ I20 (I), I996, p.5I-68. 
pour les membres de l'École, un exercice pratique dont ils sentirent vite le prix », affirme Georges Radet ${ }^{40}$.

Tandis que l'archéologie s'affirme en France comme une discipline à part entière, l'EFA contribue puissamment à l'élaboration d'une véritable méthode scientifique, inspirée par l'Altertumswissenschaft. Outre les fouilles auxquelles participent les Athéniens, Albert Dumont et Paul Foucart les initient à l'épigraphie et à l'élaboration de corpora de sources fondés sur le classement chronologique, typologique, géographique et la mise en série des vestiges recueillis lors des fouilles ou des voyages réalisés en Grèce et en Asie mineure. Au-delà de la quête de l'objet rare et beau (qui n'est cependant pas absente), il s'agit désormais de révéler l'existence des cultures anciennes et d'en écrire l'histoire. On s'appuie pour cela sur les fouilles et la publication rigoureusement organisée des sources matérielles qu'elles révèlent, en tenant compte de tous les vestiges, même les plus modestes. Dans le prolongement de cette méthode nouvelle, Albert Dumont développe la rédaction de catalogues raisonnés des collections archéologiques existantes : Léon Heuzey rappelle que «le futur directeur de l'École d'Athènes qui, plus tard, non parfois sans trouver quelque résistance, imposera à ses élèves de débuter par des catalogues détaillés et rigoureux, donne d'avance l'exemple, en se mettant lui-même à ce régime sévère. Se rencontre-t-il quelque série de monuments négligés et comme méprisés, soit parce que l'étude en est particulièrement ardue, soit parce qu'elle ne semble pas promettre des découvertes retentissantes, c'est à ceux-là que le jeune savant s'attache avec prédilection. On dirait qu'il y a de la bonté chez lui, même pour les choses. Il sait d'ailleurs que, dans ces humbles débris, résidus de la civilisation antique, on trouve souvent plus de métal précieux, plus de renseignements et de faits que n'en promet un examen superficiel. Toute la question est de savoir traiter ces scories, de les soumettre à une pression plus forte, de les fondre à une flamme plus vive ${ }^{41}$ ».

Depuis le «triennat Dumont ${ }^{42}$, telle est la conception des études qui prévaut à l'EFA. C'est donc bien à Athènes seulement que le jeune agrégé de lettres fait “ses premières armes en archéologie pratique ${ }^{43}$ ». Avant cette date en effet, rien n'indique qu'il débarque au Pirée avec la volonté de s'initier à l'archéologie. Il fait partie de ces « conscrits [qui] n'ont fait qu'un saut de l'agrégation à l'école» et qui ne sont que «l'espérance d'un bon Athénien ${ }^{44}$ ». Or il est probable qu'en raison de

40 Igor, p. IgI.

41 L. Heuzey, “Notice sur les travaux de M. Dumont», in Mélanges d'archéologie et d'épigraphie réunis par Th. Homolle, Paris, Ernest Thorin, I892, p. XI.

42 G. Radet, igoi, p. I8I.

43 A. Audollent, “Notice sur la vie et les travaux de M. Pierre Paris, membre de l’Académie », CRAI I940, р. I85.

44 G. RADET, IgOI, p. 204. 
sa formation classique, il attende avant tout de son séjour de mieux connaître l'Antiquité grecque et de se familiariser avec la beauté de ses vestiges, en prenant pour guide les auteurs anciens, selon une démarche philologique bien plus qu'archéologique qui a longtemps prévalu à l'EFA ${ }^{45}$. Le témoignage, laissé en Igor par Henri Joly quant aux raisons qui les avaient incités, lui et Louis Petit de Julleville (rappelons qu'il enseigne à l'Ens lorsque Pierre Paris y est élève) à devenir Athéniens, est ici éclairant: «Nous avions fait le projet, Petit de Julleville et moi, d'aller ensemble à l'École d'Athènes. Ce n'était pas par enthousiasme pour l'archéologie, mais nous aimions beaucoup les voyages ${ }^{46}$. " Cette démarche de pèlerin dilettante est bien éloignée de celle qui prévaut à l'EFA des années I870-I880. Mais, dans ce contexte, on peut émettre des doutes quant à l'influence qu'aurait pu avoir Petit de Julleville sur Pierre Paris dans sa conversion à l'archéologie et ce, même si Petit est, avec son condisciple Paul Decharme, le découvreur du Mouseion de l'Hélicon (qui fait l'objet de son mémoire de troisième année à l'EFA) ${ }^{47}$. La remarque vaut pour Fustel de Coulanges qui, nous dit Georges Radet, “préféra toujours les recherches de bibliothèque aux prouesses de l'archéologie conquérante. Quatre pieds carrés lui suffisaient, comme à Spinoza, pour construire un univers ${ }^{48}$ ». La démarche philologique, souvent teintée de romantisme et fondée avant tout sur l'analyse des auteurs anciens et la confrontation de leurs dires avec la réalité matérielle contemporaine, est d'ailleurs présente chez Pierre Paris. Lorsqu'il localise les ruines d'Élatée en Phocide et les vestiges du temple d'Athéna Cranaia, qu'il fouille personnellement lors de son séjour et qui seront l'objet de sa thèse de doctorat, Pierre Paris écrit: “Le temple, à n’en pas douter, était celui dont a parlé Pausanias, et qu'il appelle le hiéron d'Athéna Cranaia. [...] J'étais, je l'avoue, séduit avant tout par l'admirable situation des ruines, par le spectacle des âpres rochers d'alentour, et les lignes grandioses de l'horizon où, par delà les plaines de Phocide étalées à mes pieds, le Parnasse découpait ses trois cimes dans l'azur pur et profond du ciel. Il me semblait qu'un séjour dans ces montagnes sereines serait une succession d'heures charmantes, et que le travail, même en cas de déconvenue, porterait en lui-même sa récompense ${ }^{49}$.» Cependant, cette démarche initiale est très vite complétée par un travail de terrain répondant aux exigences fixées par Albert Dumont et Paul Foucart, un travail pour lequel les jeunes Athéniens étaient mal

45 C. VAlenti, «Le voyage en Grèce des membres de l'École française d'Athènes. Du périple héroïque à l'aventure scientifique, I846-I892 », Balkanologie 6 (I-2), 2002, p. I55-166.

46 Annuaire de l'association des anciens élèves de l'École normale supérieure Igor, cité dans C. Valenti, 2002, p. I63.

47 G. Radet, Igoi, p. I/33. Voir aussi les CRAI I866, p. I53.

48 G. Radet, I9oi, p. I23.

49 P. PAris, Élatée. La ville. Le temple d’Athéna Cranaia, Paris, Ernest Thorin, Bibliothèque des Écoles françaises d'Athènes et de Rome, fasc. 6o, I892, p. VII-VIII. 
préparés, si l'on en croit Georges Radet, arrivés à Athènes avec pour seul bagage intellectuel les rudiments enseignés par le latiniste Gaston Boissier qui leur «avait appris ce que c'était que le corpus ${ }^{50}$ ».

À cette époque en effet, les jeunes Athéniens ne reçoivent aucune formation préalable. Il faut attendre la fin du siècle et la direction de Théophile Homolle (I89o-Igo3) pour que l'on exige des nouveaux venus des connaissances méthodologiques en matière d'archéologie, d'épigraphie, de céramologie, etc., dispensées dans les grandes institutions parisiennes que sont la Sorbonne, le Collège de France, l'École du Louvre ou l'École pratique des hautes études. L'objectif affiché est alors de faire de l'école un véritable établissement scientifique, “une école d'érudition ${ }^{51}$ ». Mais lorsque Pierre Paris arrive dans la maison du Lycabette, il n'en est rien. L'EFA ne s'est pas encore transformée en « une sorte de petit Collège de France archéologique ${ }^{52}$ », elle n'est encore qu'une école de perfectionnement. Ses jeunes membres ne bénéficient pour seule propédeutique que du droit de séjourner à l'Académie de France à Rome durant quelques semaines. Nommés en octobre, ils y arrivent généralement en novembre ou décembre et séjourne à la Villa Médicis un trimestre, avant de poursuivre leur voyage vers Athènes où ils s'installent en mars ${ }^{53}$. S'ils ont alors l'opportunité de se familiariser avec les vestiges antiques, découvrant les grands sites romains et les musées de la Ville éternelle, ils ne reçoivent pas une formation d'archéologue. On s'efforce d'aiguiser leur regard artistique par «un commerce intime et prolongé » avec les artistes du Pincio, de former leur jugement, leur goût et leur sentiment du beau, à une époque ou archéologie et histoire de l'art restent intimement liées : ‘L’archéologie ne peut se passer de certaines connaissances et surtout de certaines façons de juger qui n'appartiennent qu'aux artistes ${ }^{54}$.» En somme, c'est un Pierre Paris insuffisamment préparé qui arrive à Athènes au début de l'année ı883, comme tous ses camarades qui ne sont que « des coques de noix vides ${ }^{55}$ ».

Dans ce contexte, on comprend aisément que le séjour athénien soit une période charnière dans la vie de Pierre Paris. Si Gustave Lanson a pu écrire que «toutes les vocations peuvent s'éveiller et s'armer dans la maison de la rue d'Ulm ${ }^{56}$ », il semble plutôt que ce soit la maison du Lycabette qui jette Pierre Paris sur les sentiers

50 G. Radet, I9OI, p.2I5.

51 Ibid., p.224-225. Ce désir de recevoir des jeunes gens bien préparés est l'un des objectifs de la réforme menée sous la direction de Th. Homolle. Le décret qui lui donne vie est rendu le i8 juillet I899. Le texte intégral est publié dans G. Radet, I9OI, p.442-443.

53 Ibid., p. 206.

54 Ibid., p.242.

55 Ibid., p.2I5.

56 G. Lanson, I926, p.2I. 
d'une archéologie professionnelle naissante et scelle par là même son avenir. Ce sont bien les années athéniennes qui, en lui permettant de compléter sa formation classique et sa connaissance livresque de l'Antiquité par un véritable travail de terrain et une formation méthodologique solide, lui font découvrir le métier d'archéologue. Pierre Paris fait partie de ce petit nombre de jeunes érudits qui reçoivent «la marque athénienne» et reviennent transformés par leur séjour dans “la Terre Sainte de l'Art ${ }^{57}$ ». Les différents Rapports de la Commission des Écoles d'Athènes et de Rome sur les travaux de ces deux Écoles, présentés chaque année devant l'Académie des Inscriptions et Belles-Lettres, nous permettent de suivre en partie l'activité de Pierre Paris en Grèce. Au fil des lignes rédigées par les rapporteurs ${ }^{58}$, nous assistons à sa conversion à l'archéologie, une conversion qui passe par quatre rites initiatiques.

\section{Le séjour athénien et la conversion à l'archéologie (1882-1885)}

Les étapes qui conduisent Pierre Paris à accomplir sa conversion à l'archéologie méritent chacune un développement propre que nous ne pouvons guère nous permettre ici. Nous nous contenterons d'en fixer les principaux cadres.

L'initiation au classement raisonné des sources matérielles. Selon la tradition fixée par Albert Dumont, l'une des premières tâches de Pierre Paris est de se familiariser au travail de classification des sources matérielles. Lors de la séance du 3o mai ı884, l’Académie est ainsi informée de la réception d'un mémoire de Pierre Paris transmis par Paul Foucart. Il s'agit en réalité d'un Supplément au catalogue des figurines en terre cuite du musée de la Société archéologique d'Athènes ${ }^{59}$. La multiplication des fouilles entreprises, à l'image de celles menées sur la nécropole de

57 G. RADET, I9OI, p. 422 .

58 Les rapports qui correspondent aux années pendant lesquelles Pierre Paris séjourne à Athènes sont les suivants: A.Dumont, "Rapport de la Commission des Écoles d'Athènes et de Rome sur les travaux de ces deux écoles pendant l'année ı883 ", CRAI I883, p.346-375, L.Heuzey, “Rapport de la Commission des Écoles d'Athènes et de Rome sur les travaux de ces deux écoles pendant l'année i885 », CRAI I887, p.97-IIg. Le texte de H. Weil, “Rapport de la Commission des Écoles d'Athènes et de Rome sur les travaux de ces deux écoles pendant l'année ı886 ", CRAI I887, p.595-6r6, sera consulté avec profit (il contient des informations relatives aux voyages épigraphiques que Pierre Paris fait en compagnie de Georges Radet, membre de l'efa depuis i884). Aucun rapport n'est présenté à l'Académie pour l'année I884 en raison de la mort prématurée d'Albert Dumont qui devait se charger de la présentation. "Cette lacune même sera comme une marque de deuil dans les annales des deux Écoles », écrit Léon Heuzey (I887, p. 99). CRAI I885, p. ig3. Voir également L. Heuzey, I885, p. IOo. 
Tanagra $^{60}$ (I870) ou à Myrina sous la direction d'Edmond Pottier, Salomon Reinach et Alphonse Veyries ${ }^{61}$ (I880-I882), a en effet permis de recueillir un grand nombre d'objets, notamment des figurines en terre cuite, qu'il est indispensable de cataloguer. L’ouvrage rédigé par Pierre Paris, jamais publié, vient ainsi compléter le Catalogue des figurines en terre cuite du musée de la Société archéologique d'Athènes de Jules Martha (I880) ${ }^{62}$, qui dressait une description de la collection dans son état de juillet I879. Si Léon Heuzey souligne que Pierre Paris produit un supplément “considérable et rendu nécessaire par le rapide accroissement des collections athéniennes ${ }^{63}$ ”, rien n'indique qu'il ait modifié la méthode choisie par son prédécesseur ${ }^{64}$. Martha et Paris suivent ainsi les pas de Maxime Collignon, auteur du Catalogue des vases peints du musée de la Société archéologique d'Athènes ${ }^{65}$. Quelles que soient les imperfections du Catalogue et de son Supplément, cette première activité athénienne permet au jeune Pierre Paris de se familiariser avec le travail de classification méthodologique des sources matérielles, un travail devenu indispensable en raison de la quantité de vestiges récemment découverts. S'il ne semble pas avoir été au cœur de l'activité déployée par Pierre Paris à Athènes (rappelons qu'il ne sera pas publié), il est cependant fortement valorisé par les figures d'autorité que sont Albert Dumont et Léon Heuzey. Le premier affirme ainsi qu'un «catalogue descriptif, quand il est rédigé avec méthode et avec soin, est peut-être le genre de travail qui développe le plus heureusement les qualités de l'archéologue ${ }^{66}$ ». Pour le second, “ce travail, œuvre d'observation attentive et de désintéressement scientifique, aura contribué à former son expérience et

60 Cf. V. Jeammet (dir.), Tanagra, mythe et archéologie, Paris, rmn, 2003, V. JeAmmet, La vie quotidienne en Grèce: des terres cuites pour la vie et l'au-delà, Paris, RMN-Musée du Louvre, 200I.

61 E. Pottier \& S. Reinach, La nécropole de Myrina, 2 vol., Paris, Ernest Thorin, I887. Alphonse Veyries, Athénien et ancien compagnon de Pierre Paris à l'ens (promotion de I878) participe aux fouilles mais il meurt à Smyrne le 5 décembre ı882. En ı883, l'école fait don de la collection de terres cuites de Myrina au Musée du Louvre.

62 Paris, Ernest Thorin, Bibliothèque des Écoles françaises d'Athènes et de Rome, fasc. I6, I880.

63 L. Heuzey, i887, p. Ioo.

64 Malgré nos efforts et l'aide apportée par madame Katie Brzustowski-Vaïsse, Conservateur de la bibliothèque de l'EFA, nous n'avons pu, à ce jour, localiser ce document.

65 Paris, Ernest Thorin, Bibliothèque des Écoles françaises d'Athènes et de Rome, fasc. 3, I878.

66 A. Dumont, I883, p.356. À ce sujet, Georges Radet écrit que «sur son conseil [celui d'A.Dumont], Collignon, Paul Girard, Martha, Pottier entreprirent l'inventaire des collections athéniennes, inventaire qui devait après eux occuper bien d'autres générations, les former, leur donner cette éducation technique, cette délicate 
à développer en lui les qualités d'archéologue qui se montrent aujourd'hui dans une étude tout à fait neuve et personnelle, dans une exploration accompagnée de fouilles ${ }^{67}$ „ (Heuzey évoque les fouilles menée à Élatée).

L'initiation au travail de terrain: les fouilles archéologiques. L'activité des jeunes Athéniens ne saurait toutefois se limiter à un travail de cabinet. Ils doivent aussi se familiariser avec le terrain. Les grandes fouilles que l'État grec concède à l'EFA à partir des années I87o fournissent l'occasion de les former. Le 9 mai i884, l'Académie des Inscriptions et Belles-Lettres réceptionne un mémoire rédigé par Pierre Paris intitulé Fouilles à Délos; il présente le résultat de ses recherches dans les quartiers du lac et du théâtre, menées en juillet - août i883. Il a notamment fouillé plusieurs maisons dont il a pu restituer le plan et une partie du décor (par exemple la mosaïque de la maison des Dauphins), recueillant par ailleurs un grand nombre d'inscriptions et des fragments de statues. Il retrouve Délos l'année suivante, en juillet i884, et découvre à cette occasion deux statues archaïques sur l'agora des Italiens ${ }^{68}$.

Formé sur le chantier-école de l'île sacrée d'Apollon, le jeune Athénien peut désormais se lancer dans des fouilles personnelles, une fois son projet validé par le directeur de l'École. Le ı juillet ı884, Pierre Paris fait parvenir à l'Académie, toujours par l'intermédiaire de Paul Foucart et du ministre de l'Instruction publique, un premier état des lieux des fouilles qu'il a entreprises sur le site d'Élatée en Phocide et au temple d'Athéna Cranaia ${ }^{69}$. Le I7 avril I885, c'est le mémoire des Fouilles du temple d'Athéna Cranaia qui est réceptionné par les Académiciens $^{70}$. Léon Heuzey en fera un long compte rendu devant ses pairs le 22janvier I886, soulignant la qualité des recherches menées et incitant son auteur à publier ses résultats, tout en lui prodiguant des conseils pour améliorer l'ensemble: “Après une légère révision du style, qui du reste est presque partout un bon style d'exposition, facile et simple, il y aura là, pour la Bibliothèque de l'École d'Athènes, un nouveau volume de tous points excellent ${ }^{71}$.» Ainsi encouragé, Pierre Paris en

perception des nuances, faute desquelles l'antiquaire n'est qu'un charlatan ou une dupe» (G. Radet, I9OI, p. I95).

67 L. HeuZey, I887, p. IOO.

68 CRAI I885, p. I9o. Voir également A. Dumont (I883), p.357. Nous donnons à la suite les publications de Pierre Paris correspondant à son activité dans l'île sacrée d'Apollon. Il s'agit de “Fouilles de Délos. Maisons du II siècle av. J.-C. », вCH 8, I884, p. 473-496 (pl. XX-XXI); “Inscriptions choragiques de Délos », $B C H$ 9, I885, p. I46-I57; « Statue archaïque de Délos », $в C H$ I3, I889, p. 2I7-225 (pl. VII).

69 CRAI I 885, p. 325.

70 CRAI I886, p. 93.

71 L. Heuzey, I887, p. I05. 
fera l'objet de sa thèse de doctorat, soutenue à Paris en i8gi. Le résultat de ses travaux sera publié en $1892^{72}$.

L'initiation à l'épigraphie. De surcroît, Pierre Paris participe à plusieurs voyages en Grèce et en Asie mineure (seul ou accompagné), « non pour satisfaire une vaine curiosité, mais pour y chercher des sujets d'étude et de mémoires ${ }^{73}$ », en explorant les sites antiques et en recueillant un grand nombre d'inscriptions qui seront publiées dans le Bulletin de correspondance hellénique. La démarche, encouragée depuis l'époque d'Albert Dumont, n'a rien à voir avec les voyages des premiers Athéniens, comme ceux de Charles Benoît qui «s'égarait poétiquement au milieu des ruines ${ }^{74}$ », ou ceux d'Eugène Gandar qui “consciencieusement, dans tous les lieux saints de l'Antiquité classique, [...] communi[ait] avec l'âme des grands poètes $^{75}$ ». Le dilettantisme s'est depuis effacé au profit de la méthode historique: “Nous ne saurions trop recommander aux membres de l'École d'Athènes de ne jamais passer, dans leurs voyages, devant un monument épigraphique sans en prendre une copie ou un estampage. Les textes divers d'une même inscription, sans parler de celles qui seraient inédites, sont comme les divers manuscrits d'un même ouvrage, dont la collation peut mettre sur la voie de la véritable leçon d'un passage désespéré ${ }^{76}$. Il s'agit bien ici d'une véritable formation à l'épigraphie et à ses méthodes, une formation qui, pour reprendre l'expression canonique forgée par Georges Radet, consiste en une véritable “chasse aux inscriptions ${ }^{77}$ »à

72 P. PARis, i892. Avant même de faire l'objet d'une monographie, les recherches phocidiennes de Pierre Paris sont régulièrement publiées dans la revue de l'EFA: “Fouilles à Élatée », $B C H$ 7, I883, p.518 (il s'agit d'une simple note informative); “Fouilles d'Élatée. Nouveau fragment de l'édit de Dioclétien », BCH 9, I885, p. 222-239; “ Inscriptions d'Élatée », $B C H$ Io, I886, p.356-385; “Fouilles d'Élatée. Le temple d'Athéna Cranaia », BCH II, I887, p.39-63 (pl. I, II et VI); “Fouilles d'Élatée. Inscriptions du temple d'Athéna Cranaia », $в C H$ II, I887, p.3ı8-346; “Fouilles au temple d'Athéna Cranaia. Les ex-voto », BCH II, I887, p.405-444 (pl. III, IV et V); “Fouilles au temple d'Athéna Cranaia. Catalogue des ex-voto ", $B C H$ I2, I888, p.37-63.

G. Radet, Igoi, p. I95.

G. RADET, I9OI, p. 8I.

G. RADET, Igoi, p. 90.

Le conseil est de Joseph Guignaut, rapporté dans G. RADET, IgoI, p. II6.

77 G. Radet, I9oI, p. 262. Les résultats de cette battue épigraphique sont publiés par Pierre Paris, seul ou avec ses compagnons de voyage: “Inscriptions de Sébaste », BCH 7, I883, p.448-457; “Inscriptions d'Euménia », $B C H$ 8, I884, p. 233-254; “Inscriptions de Lydie », BCH 8, I884, p.376-39o; \& M. Holleaux, “Inscriptions de Carie», $в C H$ 9, I885, p.68-84; \& M.Holleaux, “Inscriptions de Carie», $B C H$ 9, I885, p.324-348; \& G.Radet, “Deux nouveaux gouverneurs de provinces », $B C H$ 9, I885, p.433-436; \& G. RADET, “Inscriptions d'Attaleia, de Pergé, d'Aspendus », BCH IO, I886, p. I48-I6I; \& M. Holleaux, “Inscriptions d'Enoanda », 
travers la Grèce et l'Asie mineure. Délos et Élatée participent toutefois elles aussi à cette moisson épigraphique et réservent à notre jeune archéologue d'heureuses surprises, comme la découverte de plusieurs fragments des reçus de l'amende versée par les Phocidiens à l'amphictyonie delphique à la suite de la Troisième guerre sacrée (356-346 av. J.-C.) ${ }^{78}$. Ici, la démarche analogique se révèle riche d'enseignements, la confrontation des découvertes épigraphiques de Paris à Élatée avec les inscriptions de Delphes permettant de préciser l'histoire phocidienne et sa chronologie.

L'initiation au travail de publication. Chaque membre, enfin, s'initie progressivement au travail de publication en participant à la rédaction du Bulletin de correspondance hellénique. Entre i883 et I894, Pierre Paris y publie 26 articles, seul ou avec ses condisciples, Maurice Holleaux et Georges Radet (Fig. 4a). Au fil des mois et de l'expérience acquise, le nombre des publications augmente (jusqu'à 5 pour la seule année i885) ainsi que leur longueur (Fig. 4b). Si les premiers travaux restent modestes (compte rendu de lecture, actualité archéologique ${ }^{79}$ ), ils deviennent peu à peu plus complexes, prenant la forme d'articles destinés à publier les inscriptions recueillies lors de différents voyages (selon un modèle canonique imposé par Paul Foucart) ou les résultats des fouilles auxquelles les Athéniens participent. De cette manière, ils se préparent “à des ouvrages plus considérables ${ }^{80}$ » comme les mémoires adressés à l'Académie, voire même leur thèse de doctorat ${ }^{81}$. La répartition temporelle des publications témoigne par ailleurs de l'effort de l'école pour publier le résultat des travaux menés le plus rapidement possible. Ainsi, le bilan de la campagne de fouille dirigée par Pierre Paris à Délos au cours de l'été i883 est publié dans les volumes de i884 et I885. Entre I885 et I888, six articles rendent

et d'Isaurie », $B C H$ IO, I887, p.50o-5ı/; \& G. Radet, “Inscriptions de Pisidie, de Lycaonie et d'Isaurie », $B C H$ II, I887, p.63-70; \& G. RADET, «Inscriptions hypothécaires d'Amorgos », $B C H$ I3, ı889, p.342-345; \& G. Radet, “Inscriptions de Syllion en Pamphylie», BCH I3, I889, p.486-497; \& G. Radet, “Inscription relative à Ptolémée, fils de Thraséas », $B C H$ I4, I89o, p.587-589; enfin, “Inscriptions de Phocide et de Locride», $B C H$ I8, I894, p.53-63. Mentionnons également la thèse latine préparée par Pierre Paris en vue d'obtenir son doctorat, en complément de sa thèse principale sur Élatée. Elle s'appuie en effet sur ses travaux épigraphiques en Asie mineure: Quatenus feminae res publicas in Asia minore, Romanis imperantibus, attigerint, Paris, Ernest Thorin, I892 (soutenue en I89I). P. PARIs, “Fouilles d'Élatée. Inscriptions », I887, p.32I.

79 P. PARIs, «Fouilles », I883, p.5ı8, ainsi que «Bibliographie. Étude sur les lécythes blancs attiques à représentations funéraires, par E. Pottier », $B C H$ 8, I884, p.223-224.

80 A. Dumont, I883, p. 346-347.

81 Ainsi, les six articles (et les planches qui les accompagnent) que Pierre Paris publie dans le Bulletin sur ses fouilles à Élatée constitueront la base de la monographie qui paraîtra en I8g2. 
compte des fouilles menées à Élatée en ı884. En revanche, les publications qu'entraîne la “chasse aux inscriptions » s'étalent davantage dans le temps : six articles sont publiés jusqu'en i885, date à laquelle Pierre Paris rentre en France. Jusqu'en I894, neuf autres suivent: pour les inscriptions de Phocide et de Locride, objet de l'article paru en ı894, près de dix ans s'écoulent entre leur découverte et leur publication. Concernant la statue découverte sur l'agora des Italiens à Délos (juillet I884), son étude n'est publiée dans le Bulletin que cinq ans plus tard, en i889. Ces retards, déplorés par Georges Radet dans sa monographie sur l'histoire de l'école, s'expliquent sans doute par l'importance numérique des découvertes, la périodicité du Bulletin (un numéro par an seulement), ainsi que par la méthode exhaustive exigée par Paul Foucart pour la publication des inscriptions ${ }^{82}$.

\section{"Et la Grèce le scella de son empreinte ${ }^{83}$ »}

La revue de l'EFA est donc en partie le reflet des quatre rites initiatiques que les jeunes Athéniens doivent accomplir pour pouvoir prétendre au titre d'archéologue. Transformées en pourcentages, les données que nous avons recueillies ne font que confirmer ce que l'on pressentait (Fig. 5a et 5b): les travaux de Pierre Paris font la part belle à l'épigraphie, entre $49 \%$ et $58 \%$ du total, selon que l'on envisage le nombre de pages ou le nombre de publications consacrées aux inscriptions recueillies, pour l'essentiel, en Asie mineure (leur commentaire appelant un développement plus ou moins long selon l'importance de la découverte). Rien de surprenant si l'on songe que Georges Radet a caractérisé cette période de l'histoire de l'école comme une ère de «renaissance épigraphique ${ }^{84}$ ». Viennent ensuite les travaux consacrés aux fouilles personnelles que Pierre Paris mène à Élatée et au temple d'Athéna Cranaia (en Phocide). Si la part du nombre d'articles est plus modeste $(23 \%)$, celle du nombre de pages représente près de $40 \%$ du total : les articles sont moins nombreux mais plus denses, reflétant l'importance des découvertes réalisées sur un territoire jusque-là peu exploré, exception faite de Delphes. Enfin, environ II \% des publications de Pierre Paris dans le Bulletin de correspondance hellénique sont consacrées à Délos. Chantier-école de l'École française d'Athènes, l'île d'Apollon voit se succéder les différentes promotions d'Athéniens et des archéologues plus chevronnés. Pierre Paris n’est donc pas le seul à y travailler: Amédée Hauvette y conduit des fouilles en I88I, Salomon Reinach en I882,

G. RADET, I9OI, p.387.

En référence à G. RADET, I9OI, p. 422.

84 G. RADEt, IgoI, p.203-2I8 (il s'agit d'un titre de chapitre consacré à la direction de Paul Foucart, entre I878 et I89o). 
Théophile Homolle et Félix Dürrbach en I885². La part des articles que Pierre Paris consacre à Délos est donc plus réduite, illustrant ce qu'est l'île sacrée: un chantier partagé.

Quoi qu'il en soit, c'est bien un archéologue qui revient en France en i885, un savant formé selon les nouvelles méthodes impulsées par Albert Dumont et Paul Foucart, familiarisé avec le travail de cabinet comme avec le travail de terrain, accoutumé aux études épigraphiques et archéologiques auxquelles il s'est consacré pendant près de trois ans. La qualité de son parcours athénien est d'ailleurs reconnue par l'Académie des Inscriptions puisque lors de la séance du i5 mai ı885, ses membres proposent Pierre Paris comme candidat à la médaille que la Société centrale des architectes accorde chaque année à un membre des Écoles de Rome ou d'Athènes, précisant qu'il « a paru mériter cette distinction par les fouilles intéressantes qui ont dégagé les restes du temple d'Athéna Cranaia, à Élatée, dans l'ancienne Phocide ${ }^{86}$ ).

L'étude de ce parcours athénien, présenté succinctement, méritera d'être développé. Mais nous pouvons d'ores et déjà affirmer que c'est bien le séjour dans la maison du Lycabette, bien plus que celui de la rue d'Ulm, qui marque un tournant décisif dans la vie de Pierre Paris. C'est bien la Grèce qui, pour reprendre une belle formule de Georges Radet, le «scelle de son empreinte ${ }^{87}$ » et fait de lui un véritable archéologue. Cette « marque athénienne ${ }^{88}$ » ou, pour utiliser un concept plus moderne, ce réseau athénien, va d'ailleurs influencer durablement (et rapidement) la trajectoire professionnelle de Pierre Paris. L'expression «carrières athéniennes » qu'utilise Catherine Valenti dans l'étude que nous avons déjà citée ${ }^{89}$ s'applique parfaitement au cas de Pierre Paris. Rentré en France en I885, il obtient, dès l'automne de cette même année, un poste à l'université de Bordeaux. En novembre, il est en effet chargé d'un cours complémentaire de langues et littératures grecques. Sans doute ne s'agit-il pas d'archéologie: la chaire d'antiquités grecques et latines

85 A. Plassart, “Un siècle de fouilles à Délos », вCH. Études déliennes suppl. I, r973, p.5-ı6. Voir également Тн. НомоLle, "Rapport sur une mission archéologique dans l'île de Délos », Archives des missions scientifiques et littéraires i3, I887, p.389-435.

CRAI I886, p.98. Son condisciple, Maurice Holleaux, n'est pas oublié, loin s'en faut. Les résultats qu'il a obtenus en fouillant le sanctuaire d'Apollon Ptoios ont convaincu les responsables de l'École de lui accorder le droit de passer une année supplémentaire (soit quatre ans) à Athènes. Voir à ce sujet H.WeIL, I886, p.595-6ı6. Si l'on en croit Georges Radet (I9oI, p. 209), “les six campagnes qu'il dirigea sur le Ptoïon le couvrirent de lauriers".

87 G. RADET, I9OI, p. 422.

88 L'expression est encore une fois de G. RADET, I9oI, p.422, passé maître dans l'art de la formule.

89 “Les membres de l'école», p. I67. 
est alors occupée par Maxime Collignon (qui rejoindra bientôt la Sorbonne). Mais dès l'année suivante, Pierre Paris est nommé maître de conférence pour les antiquités grecques et latines (7août I886), avant de devenir le premier titulaire de la chaire d'archéologie et d'histoire de l'art créée à Bordeaux en I892 ${ }^{90}$. Il fait ainsi partie des Athéniens qui obtiennent directement un poste dans le supérieur sans enseigner dans un lycée, dans un contexte bien particulier, celui de la réforme universitaire des années I880-I8go à la faveur de laquelle les facultés et les chaires se multiplient. Fortement spécialisés et dûment qualifiés, nombreux sont les Athéniens à bénéficier de ce processus qui leur ouvre rapidement les portes de l'enseignement supérieur et de la recherche ${ }^{91}$. C'est donc autour de «la petite colonie athénienne de Bordeaux ${ }^{92}$ » que se déroulera le second acte de la carrière de Pierre Paris.

\section{Grégory Reimond}

Université Toulouse-Jean Jaurès

PLH-ERASME (EA 4601)

gregoryreimond1984@gmail.com

90 Annuaire des facultés de Bordeaux. I893-1894, Bordeaux, Imprimerie de l'Académie et des facultés, I893, p. I4.

91 C. Valenti, «Les membres de l'école», p. i67-I7ı.

92 G. Radet, I9OI, p.378. 
Fig. I. L'acte de naissance de Pierre Paris

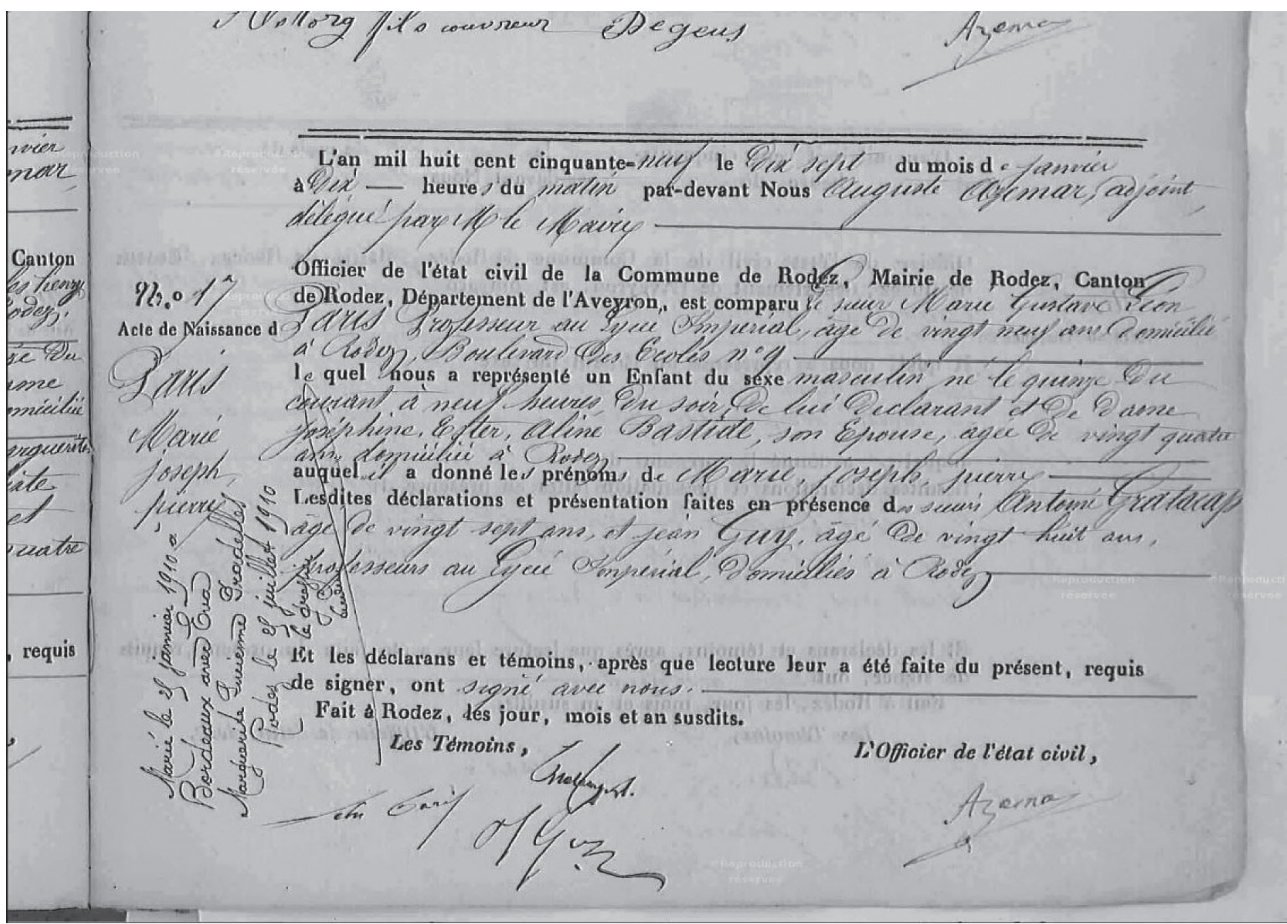

Source: Archives départementales de l'Aveyron: 4E2I2-38 RODEZ NA ı859/1859 (oI/OI/I859 - 28/oi/1859).

Fig. 2. L'origine géographique et sociale des Athéniens (I846-I990) Les cases grisées correspondent au profil de Pierre Paris

\begin{tabular}{|lc|}
\hline \multicolumn{2}{|c|}{ Origine géographique (\%) } \\
\hline Nord-Est & 28 \\
\hline Paris et sa région & 27 \\
\hline Nord-Ouest & 14 \\
\hline Sud-Ouest & 8 \\
\hline Centre & 7 \\
\hline Sud-Est & 4 \\
\hline $\begin{array}{l}\text { Né dans un chef-lieu de } \\
\text { département de province }\end{array}$ & 24 \\
\hline Nés à l'étranger & 3 \\
\hline
\end{tabular}

\begin{tabular}{|lc|lc|}
\multicolumn{5}{|c|}{ Origine sociale (\%) } & 5 \\
\hline Professeurs du secondaire & 22 & Ingénieurs & 4 \\
\hline Médecins / pharmaciens & 11 & Officiers & 3 \\
\hline Universitaires & 9 & Artistes & 2 \\
\hline Commerçants & 9 & Instituteurs & 2 \\
\hline Professions juridiques & 9 & Employés & 2 \\
\hline $\begin{array}{l}\text { Fonctionnaires autres } \\
\text { qu'enseignants }\end{array}$ & 7 & Industriels & \\
\hline Propriétaires / rentiers & 7 & Banquiers / assureurs & 2 \\
\hline Veufs / veuves & 6 & Ouvriers & 0 \\
\hline
\end{tabular}

Source : élaboration personnelle d'après C. VALENTI, «Les membres de l'École française d'Athènes : étude d'une élite universitaire (I846-ı992) », BCH I20 (I), I996, p. I57-I72. 
Fig. 3. De l'Ens aux Écoles françaises d'Athènes et de Rome:

les prémices d'un réseau savant?

\begin{tabular}{|c|c|c|c|c|}
\hline & Promotion École française & \multicolumn{2}{|c|}{ Promotion ENS Ulm } & Promotion École française de Rome \\
\hline 1878 & Hauvette Amédée & 1875 & $\begin{array}{c}\mathrm{X} \\
\mathrm{X} \\
\mathrm{X} \\
\mathrm{X} \\
\mathrm{X} \\
\mathrm{X} \\
1874\end{array}$ & $\begin{array}{l}\text { CuQ Édouard } \\
\text { DELAVILLE-LEROULX Joseph } \\
\text { DONAT Charles } \\
\text { DUCOUDRAY-LA BLANCHĖRE René } \\
\text { DURRIEU Paul } \\
\text { ENGEL Arthur } \\
\text { LAFAYE Georges }\end{array}$ \\
\hline 1879 & $\begin{array}{l}\text { DuboIs Marcel } \\
\text { REINACH Salomon }\end{array}$ & $\begin{array}{l}1876 \\
1876\end{array}$ & $\begin{array}{l}1876 \\
X \\
X\end{array}$ & $\begin{array}{l}\text { LACOUR-GAYET Georges } \\
\text { MARTIN Albert } \\
\text { THOMAS Antoine }\end{array}$ \\
\hline 1880 & $\begin{array}{l}\text { BARRILLEAU Georges } \\
\text { BILCo Joseph } \\
\text { CleRC Michel }\end{array}$ & $\begin{array}{c}X \\
1877 \\
1877\end{array}$ & $\begin{array}{c}X \\
1877 \\
X\end{array}$ & $\begin{array}{l}\text { FauCon Maurice } \\
\text { JuLliAn Camille } \\
\text { VigneauX Émile }\end{array}$ \\
\hline 1881 & $\begin{array}{l}\text { ENGEL Arthur } \\
\text { MONCEAUX Paul } \\
\text { VEYRIES Alphonse }\end{array}$ & $\begin{array}{c}\mathrm{X} \\
1878 \\
1878\end{array}$ & $\begin{array}{c}1878 \\
X \\
1878\end{array}$ & $\begin{array}{l}\text { DIEHL Charles } \\
\text { GRANDJEAN Charles } \\
\text { SALOMON Charles }\end{array}$ \\
\hline 1882 & $\begin{array}{l}\text { HolleauX Maurice } \\
\text { Paris Pierre }\end{array}$ & $\begin{array}{l}1879 \\
1879\end{array}$ & $\begin{array}{c}\mathrm{X} \\
1879 \\
1879 \\
\mathrm{X} \\
\mathrm{X}\end{array}$ & $\begin{array}{l}\text { DiGARD Georges } \\
\text { FABRE Paul } \\
\text { GrousSET René } \\
\text { NOLHAC Pierre de } \\
\text { PoISNEL-LANTILLIĖrE Charles }\end{array}$ \\
\hline 1883 & $\begin{array}{l}\text { CousIN Georges } \\
\text { DIEHL Charles } \\
\text { DÜRRBACH Félix }\end{array}$ & $\begin{array}{l}1880 \\
1878 \\
1880\end{array}$ & $\begin{array}{c}\mathrm{X} \\
1880\end{array}$ & $\begin{array}{l}\text { LANGLOIS Ernest } \\
\text { LÉCRIVAIN Charles }\end{array}$ \\
\hline 1884 & RADET Georges & 1881 & $\begin{array}{c}\mathrm{X} \\
1881 \\
\mathrm{X}\end{array}$ & $\begin{array}{l}\text { BERTHELOT André } \\
\text { PÉrATÉ André } \\
\text { Prou Maurice }\end{array}$ \\
\hline
\end{tabular}

Source : élaboration personnelle à partir de l'annuaire des anciens élèves, élèves et amis de l'Ens (http://www.archicubes.ens.fr) et des listes des anciens élèves de l'EFR (http:/www.ecole-francaise.it/fr/PDF/AnnuaireEFR.pdf) et de l'EFA (http://www.EFA.gr/Ecole/Histoire/ecole_hist_amb.htm) 
Fig. 4a. Nombre de publications de Pierre Paris dans le BCH par type d'activité

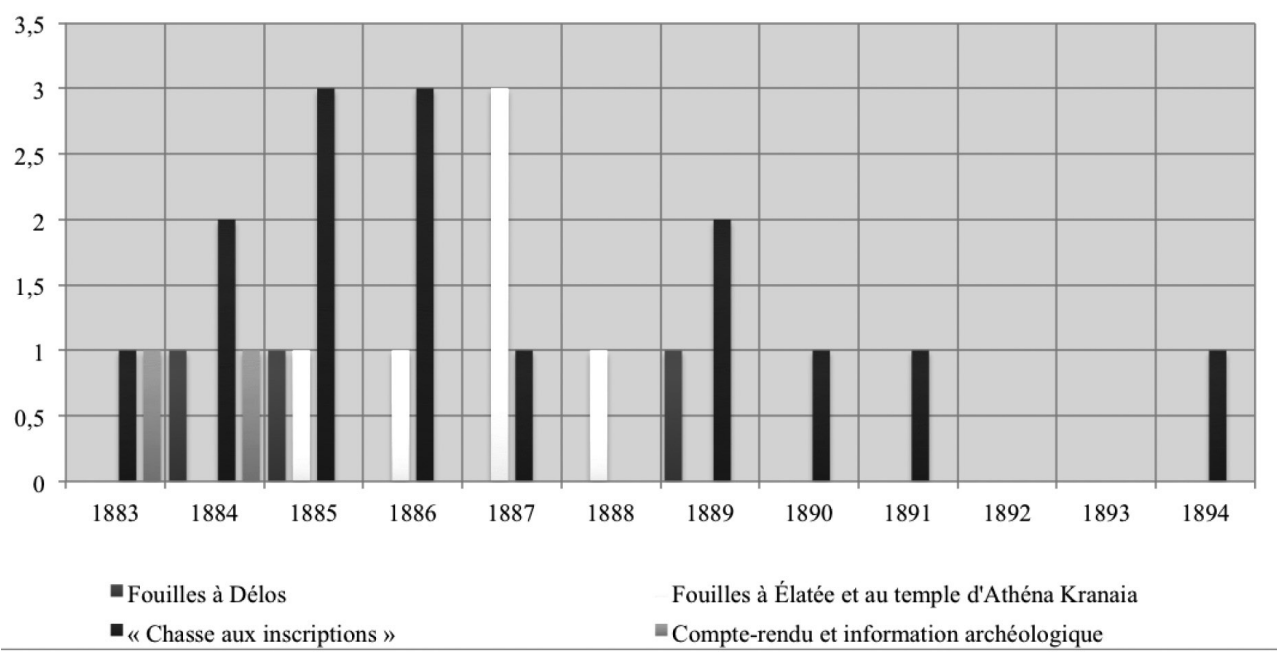

Source : élaboration personnelle d'après le Bulletin de correspondance hellénique (I883 à I894)

Fig. 4b. Publications de Pierre Paris dans le BCH.

Pagination des articles publiés par type d'activité

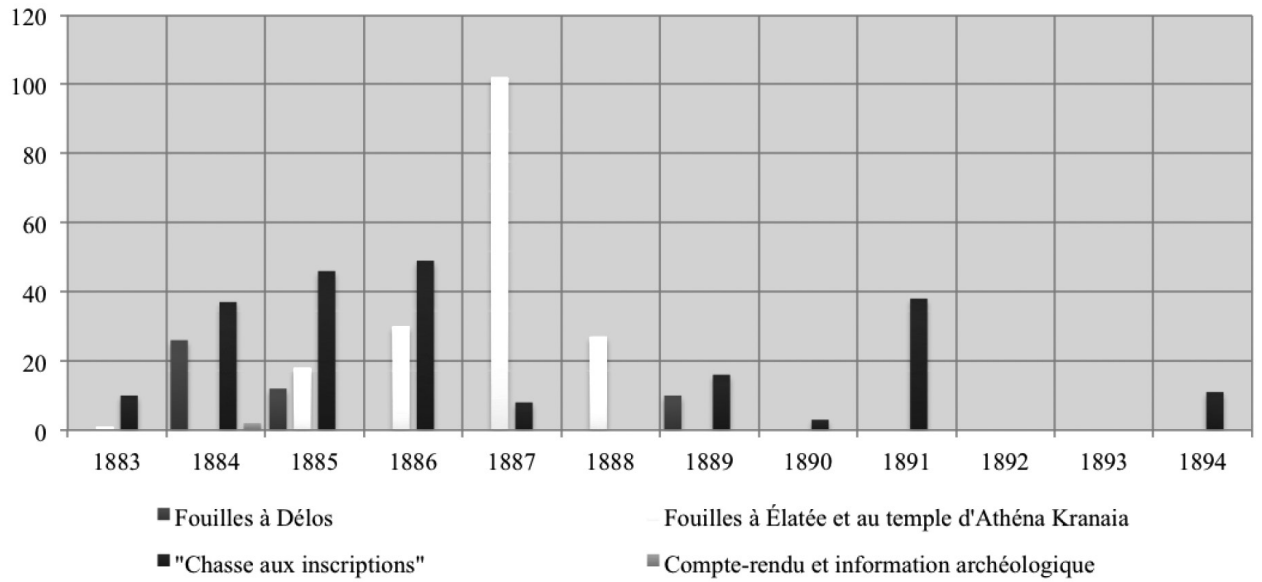

Source : élaboration personnelle d'après le Bulletin de correspondance hellénique (I883 à I894) 
Fig. 5. Les publications de Pierre Paris dans le $B C H$, reflet de son activité

Fig. 5a. Pagination par type d'activité (en \%)

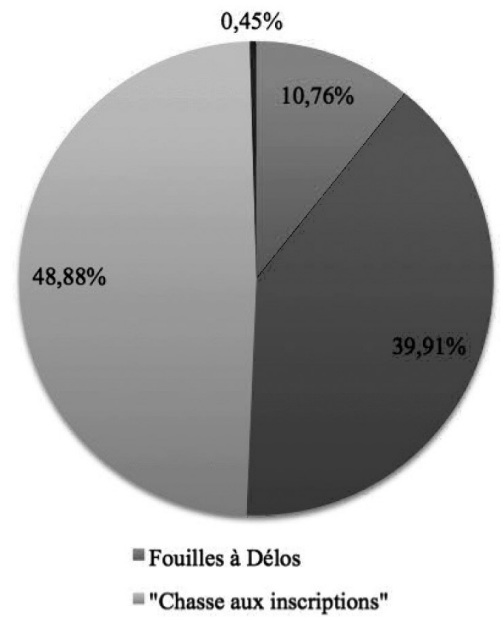

Fig. 5b. Nombre de publications par type d'activité (en \%)

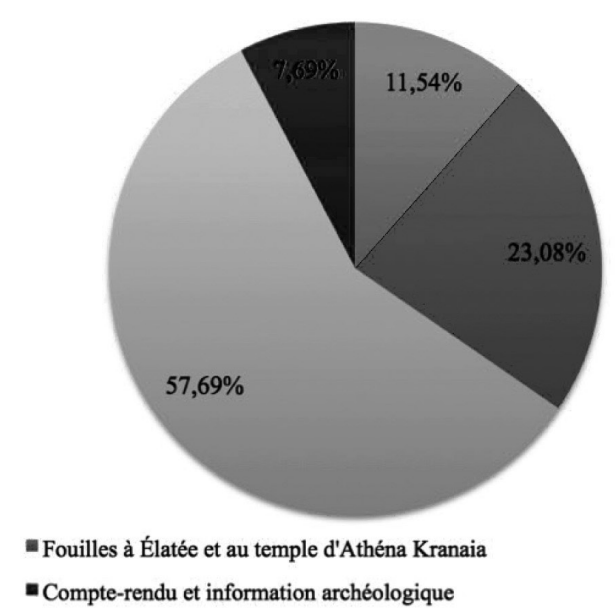

Source : élaboration personnelle d'après le Bulletin de correspondance hellénique (I883 à I894) 